\title{
BIOÉTICA Y JUSTICIA AMBIENTAL: EL CASO DE PRESENCIA DE PLAGUICIDAS EN ESCOLARES DE COMUNIDADES RURALES
}

\author{
María Teresa Muñoz-Quezada ${ }^{1}$, Boris Andrés Lucero Mondaca ${ }^{1}$ \\ Resumen: Una serie de estudios evidencian las consecuencias en la salud de las personas expuestas a plaguicidas, ya sea \\ por la actividad laboral como por vivir o estudiar cerca de predios agrícolas. La exposición ocupacional a una serie de \\ insecticidas, herbicidas y fungicidas está asociada principalmente a cáncer, daño neurocognitivo y motor, polineuropatía \\ periférica, depresión y ansiedad, malformaciones en niños al nacer, dificultades endocrinas y en el sistema reproductivo, \\ daño renal y genotóxicos. A través de una revisión de la literatura, el objetivo del siguiente artículo consistió en dilucidar \\ la situación de exposición a plaguicidas en comunidades escolares rurales y aplicar elementos de la bioética y justicia am- \\ biental para proponer un cambio y acciones que permitan mejorar su calidad de vida y salud, por el derecho a vivir libres \\ de contaminación ambiental. La justicia ambiental busca mucho más que la equidad y el tratamiento justo y de calidad \\ similar al de otras comunidades no vulnerables, apunta a hacer que las mismas comunidades exijan la necesidad de una \\ sociedad más igualitaria que vele por el derecho a vivir en un medio ambiente limpio.
}

Palabras clave: exposición a plaguicidas, justicia ambiental, escolares rurales, principio precautorio

Bioethics and environmental justice: the case of the presence of pesticides in schoolchildren in rural communities

Abstract: A series of studies show the health consequences of people exposed to pesticides either because of their work activity or because they live or study near agricultural land. Occupational exposure to a series of insecticides, herbicides and fungicides is mainly associated with cancer, neurocognitive and motor damage, peripheral polyneuropathy, depression and anxiety, malformations in children at birth, endocrine and reproductive system difficulties, kidney damage and genotoxic effects. Through a review of the literature, the objective of the following article was to elucidate the situation of exposure to pesticides in rural school communities, unveil the bioethical dilemmas in which they are located and the need to apply elements of environmental justice to generate a change and actions to improve their quality of life and health, for the right to live free of environmental pollution. Environmental justice seeks much more than equity and fair treatment and quality similar to that of other non-vulnerable communities, aims to make the same communities demand the need for a more egalitarian society that ensures the right to live in an environment cleansed.

Key words: Exposure to pesticides, environmental justice, rural schoolchildren, precautionary principle

\section{Bioética e justiça ambiental: o caso da presença de pesticidas em escolares de comunidades rurais}

Resumo: Uma série de estudos evidenciam as conseqüências na saúde das pessoas expostas a pesticidas, seja por atividade de trabalho como por viver ou estudar perdo de prédiso agrícolas. A exposição ocupacional a uma série de inseticidas, herbicidas e fungicidas está associada principalmente a câncer, dano neurocognitivo e motor, polineuropatia periférica, depressão e ansiedade, mal formações em crianças ao nascer, dificuldade endócrinas e no sistema reprodutivo, dano renal e genotóxicos. Através de uma revisão da literatura, o objetivo do artigo seguinte consistiu em elucidar a situaçáo de exposição a pesticidas em comunidades escolares rurais e aplicar elementos de bioética e justiça ambiental para propor uma mudança e açóes que permitam melhorar sua qualidade de vida e saúde, pelo direito a viver livre de contaminação ambiental. A justiça ambiental busca muito mais que a equidade e o tratamento justo e de qualidade similar ao de outras comunidades não vulneráveis, tenta fazer que as mesmas comunidades exijam a necessidade de uma sociedade mais igualitária que vele pelo direito a viver em um meio ambiente limpo.

Palavras chave: exposição a pesticidas, justiça ambiental, escolares ruraiss, princípio da precaução

\footnotetext{
${ }^{1}$ Centro de Investigación en Neuropsicología y Neurociencias Cognitivas, Facultad de Ciencias de la Salud, Universidad Católica del Maule, Chile Correspondencia:mtmunoz@ucm.cl
} 


\section{Introducción}

Actualmente, la deriva ambiental por la aplicación de plaguicidas ha generado diversos efectos adversos en la salud de las personas, contaminación de aguas, aire, suelos, especies animales, vegetales y ecosistemas, producto del escaso control y medidas preventivas respecto de la aspersión de la gran variedad de agrotóxicos que se aplican en la agricultura $(1,2)$.

Investigaciones documentan las consecuencias en la salud de niños, adolescentes y adultos expuestos a plaguicidas, ya sea por la actividad laboral como por vivir o estudiar cerca de predios agrícolas. Se ha reportado que la exposición ocupacional a una serie de insecticidas, herbicidas y fungicidas está asociada principalmente a cáncer de próstata, cáncer al pulmón, melanoma y leucemia(3). Otros estudios, encontraron efectos adversos en los niveles neurocognitivo y motor(4), polineuropatía periférica(5), ansiedad(6), malformaciones(7), disrupción endocrina(8) y del sistema reproductivo(9), daño $\operatorname{renal(10)~y~}$ genotoxicidad(11).

Por otro lado, el cambio climático ha provocado el aumento de temperaturas, insectos más resistentes a condiciones adversas y la presencia de plagas invasoras que antes no existían, lo que produce que muchos agricultores sobreexcedan el uso de plaguicidas, sin recibir la capacitación y control adecuado de su uso(12).

En diversos países se han hallado residuos de plaguicidas en frutas y verduras, dando cuenta de hasta 5 tipos de pesticidas(13). En Argentina, 6 de 10 frutas poseen más de un agroquímico, en Chile más de 2 tipos de pesticidas altamente peligrosos se han encontrado en frutas $y$, además, diversos fungicidas y preservantes(14).

En Chile se vende, en collares de animales domésticos y en productos que controlan plagas de pulgas y garrapatas(15), plaguicidas que en Europa están prohibidos, restringidos en USA o que la IARC (International Agency for Resarch on Cancer) tiene clasificados en el grupo $2 \mathrm{~A}$ como probablemente cancerígeno, siendo un ejemplo el diazinón(16). Por lo tanto, toda la población está en cierta medida expuesta y, la- mentablemente, las normativas chilenas regulan parcialmente la venta y manipulación de estos plaguicidas; cualquier adulto puede aplicarlos sin capacitación, independiente de su peligrosidad, lo que implica que el riesgo a la salud de las personas sea mayor.

Sin embargo, las poblaciones más vulneradas son las comunidades rurales, quienes aplican al interior de los hogares insecticidas que se utilizan en la agricultura, y viven, estudian y trabajan cerca de predios agrícolas que aplican una diversidad de pesticidas sin las regulaciones correspondientes (17).

El siguiente trabajo tiene como objetivo dilucidar la situación de exposición a plaguicidas en comunidades escolares rurales, y aplicar elementos de la bioética y justicia ambiental para proponer un cambio y acciones que permitan mejorar su calidad de vida y salud.

La relevancia de esta revisión se enmarca en generar un análisis reflexivo de la condición de justicia ambiental que requieren las comunidades rurales expuestas a agrotóxicos, con el fin de orientar las acciones para que se generen los espacios y las normativas que permitan que las comunidades estén protegidas y puedan participar de manera activa en las políticas que se gestionan sobre la contaminación ambiental y el uso de plaguicidas en el territorio rural.

\section{Método}

Para realizar esta revisión, se consultó la literatura científica de los últimos 15 años en las bases de datos electrónicas PubMed y SciELO, libros de divulgación científica, normativas y leyes, páginas web de instituciones y artículos vinculados a plaguicidas en escolares y a bioética y justicia ambiental. Se analizaron los contenidos de los documentos y la información se organizó en tres temáticas relevantes: a) exposición a plaguicidas en niños; b) plaguicidas y efectos en la salud de escolares, y c) situación de la exposición a plaguicidas en escolares rurales chilenos. Finalmente, la discusión aborda la evidencia desde una mirada desde la bioética y justicia ambiental. 


\section{Resultados}

\section{a) Exposición a plaguicidas en niños}

Diversos estudios internacionales demuestran presencia de metabolitos de plaguicidas en niños(17-27). La dieta se identifica como la vía de exposición dominante $y$, en segundo lugar, la aplicación de plaguicidas dentro del hogar. El mayor riesgo se observa en niños, debido a la mayor inhalación y la tasa de ingestión de vegetales por peso corporal en relación con los adul$\operatorname{tos}(17)$.

En un estudio realizado con 203 niños(18) de 4 a 6 años en EE.UU., se encontró concentraciones de metabolitos dialquilfosforados (DAPs) en la orina (asociados a pesticidas organofosforados) superiores a lo esperado para su edad. Además, se observó que las concentraciones de metabolitos eran más elevadas en niños que vivían en hogares en que se había aplicado plaguicidas al interior del domicilio con anterioridad a cuatro semanas $(\mathrm{p}<0.05)$.

Otra investigación, realizada en menores de 15 años de comunidades agrícolas en México(19), analizó 281 muestras de orina. En el 100\% de las muestras se detectaron al menos dos pesticidas de los 17 reportados en el total. La presencia de malatión, metoxurón y glifosato se encontró en más del $70 \%$ de los casos.

En un estudio para medir la exposición a plaguicidas OP a través de la dieta(20), se cambió gran parte de la dieta con productos de origen orgánico a escolares durante 5 días consecutivos y se recogió diariamente las muestras de orina durante 15 días. Se determinó que la concentración urinaria promedio de metabolitos para el malatión y clorpirifos descendió hasta niveles no detectados después del consumo de dietas orgánicas.

Por otro lado, en 89 niños de 3 y 7 años en Canadá(21) se constató la presencia de altos niveles de metabolito OP en orina de los niños. Se encontró diferencias significativas respecto del contacto con animales dentro de la casa $(\mathrm{p}=0.02)$.

Otro estudio(22) encontró concentraciones de clorpirifos más elevado en el polvo doméstico de casas donde los padres son agricultores. También, se ha reportado(23) que niños entre 2 y 5 años estaban expuestos a nivel alto de azinfos metil en el polvo doméstico. Además, niveles detectables de clorpirifos y azinfos metil en las manos y juguetes de niños cuyos padres son agricultores.

Otro estudio en California(24) reportó riesgo en la inhibición de la acetilcolinesterasa provocada por OP en niños y adultos. Se identificó la dieta como la vía de exposición dominante en la exposición acumulada. La tendencia temporal y la variación espacial se asociaron con la exposición con agua potable contaminada y el aire.

En Costa Rica se midió en 140 niños(25), que vivían cerca de plantaciones de plátanos, bananas y de granjas orgánicas, presencia de metabolitos urinarios de clorpirifos, mancozeb y piretroides (3-PBA). Las concentraciones de clorpirifos fueron más elevadas en los niños que vivían cerca de las plantaciones de plátanos y bananos, y cuyas madres tenían menos nivel educacional. Las concentraciones de mancozeb fueron mayores en niños que vivían en la aldea bananera. Los niños más pequeños y que vivían en la aldea de plátanos presentaban concentraciones mayores de piretroides.

Finalmente, un estudio realizado en el Valle de Salinas, en California(26), que reclutó a 601 mujeres embarazadas a quienes se les recolectaron muestras de orina durante el embarazo para medir metabolitos OP, encontró que, a mayor presencia de metabolitos OP prenatales, se observaban mayores rasgos relacionados con un bajo comportamiento social asociado al autismo.

\section{b) Plaguicidas y efectos en la salud de escolares}

Los efectos de la exposición a plaguicidas son similares en niños y adultos; sin embargo, sus consecuencias en el organismo van a estar vinculadas con la edad, características fisiológicas y madurez del organismo, además de las características de componente activo y categoría toxicológica del pesticida, dosis, combinación de la exposición aguda y/o crónica, y variables ambientales(27). 
Algunas investigaciones evidencian asociación positiva entre la exposición a plaguicidas y la aparición de malformaciones congénitas: defectos en el tubo neural, anomalías musculoesqueléticas, defectos en el aparato urogenital y problemas cardiovasculares en humanos(28).

En México (29) se evaluó la relación entre exposición a plaguicidas y malformaciones en 1.149 recién nacidos de comunidades que cultivan flores, en contraste con 5.069 niños urbanos. Se observó que $20 \%$ de los niños nacidos en comunidades que cultivaban flores y expuestas a plaguicidas tenían malformaciones congénitas.

Otros estudios $(30,31)$ midieron los efectos en el desempeño neuroconductual en una cohorte de hijos de madres trabajadoras agrícolas del Valle de Salinas, en California, desde la etapa prenatal. Demostraron que la exposición prenatal a OP está asociada negativamente con el desarrollo cognitivo y neuroconductual de los niños, encontrando efectos a partir de los 12 meses y continuando en la niñez temprana y escolar.

Sin embargo, en el caso de niños expuestos permanentemente a dosis bajas o moderadas también se puede observar consecuencias que se esperan que aparezcan en adultos, como el cáncer. En un estudio de caso y control realizado en Brasil(32), con 252 niños con leucemia y 423 controles, se encontró asociación con el uso de plaguicidas durante el embarazo para la leucemia linfoide aguda (LLA) y para la leucemia mieloide aguda (LMA). La exposición materna a permetrina presentó mayor riesgo de presentar un hijo con leucemia. Al mismo tiempo, la exposición a plaguicidas durante el embarazo mostró un riesgo de hasta 5 veces de presentar un hijo con LLA y de 7 veces de presentar LMA.

Resultados similares se han observado en China(33), donde se desarrolló un estudio con 390 niños con leucemia y 813 controles, encontrando que la incidencia de leucemia y la aplicación al interior del hogar de plaguicidas durante el embarazo fue 1.8 veces mayor y la asociación entre leucemia y exposición a plaguicidas en el jardín durante el embarazo fue de 2.3 veces mayor que los controles.

\section{Situación de la exposición a plaguicidas en es- colares rurales chilenos}

En los últimos años existe evidencia de que los escolares, principalmente de comunidades rurales, están fuertemente expuestos a una gama de plaguicidas y entre ellos altamente peligro$\operatorname{sos}(13,17,34-39)$.

Rojas et al.(34) reportan que un 19,11\% de nińos nacidos con malformaciones viven cercanos a predios agrícolas. Otro estudio(35), realizado en escuelas municipales de la provincia de Talca, encontró que existe 4.24 veces más probabilidad de encontrar escolares con DI en escuelas cercanas a predios agrícolas. Otra investigación relaciona el consumo de fruta y verduras con residuos de OP y la cercanía de viviendas a predios agrícolas de escolares con presencia de metabolitos OP en la orina(17).

Por otro lado, se encontró en trabajadores de la VIII región que la exposición ocupacional a mezclas de pesticidas provoca daño citogenético(36). En esta misma línea, Contreras-Levicow et al.(37) encontraron que el porcentaje de mujeres con abortos espontáneos en Llay Llay (V Región) y que posee ocupación agrícola es mayor.

Zúñiga et al.(38) hallaron que temporeras agrícolas de la VIII Región poseen mayor daño citogenético, problemas reproductivos, problemas de fertilidad, complicaciones en el embarazo, abortos espontáneos y malformaciones congénitas.

El estudio de Muñoz et al.(39), con 25 escolares rurales, encontró que un 56\% presentaba concentraciones por sobre el Límite de Detección (LD) de metabolito de OP dimetilfosfato (DMP). 92\% presentaba concentraciones sobre el LD de dimetiltiofosfato (DMTP) y $92 \%$ de los escolares presentaba sobre el LD el metabolito dietilfosfato (DEP). Las concentraciones de DMTP se asociaron con menor desempeño cognitivo en la velocidad de procesamiento de la información de los escolares $\left(r_{s}=-.44, p=.014\right)$.

Por otro lado, existen estudios de monitoreo y control ambiental de los niveles de residuos de 
plaguicidas en vegetales en Chile(40-42). Revisando los estudios que evaluaron la fruta y verdura que comen los escolares en Chile $(13,17)$, los resultados se encontraban dentro de lo esperado, según las normas técnicas del Servicio Agrícola Ganadero, SAG(40) y el Ministerio de Salud, MINSAL(41). Sin embargo, estas mediciones se realizan principalmente en empresas grandes, que poseen una alta fiscalización. La situación de huertos o predios agrícolas familiares son poco pesquisados. Un estudio reciente de la Agencia Chilena para la Calidad e Inocuidad Alimentaria, ACHIPIA(42), reveló que los compuestos químicos en alimentos más notificados son los plaguicidas, donde la hortaliza lechuga y el organofosforado metamidofos se presenta por sobre el límite permitido, poniendo en riesgo la salud de las personas. Estas irregularidades se observan principalmente en las hortalizas de consumo de la zona norte y centro de Chile. La tabla 1 muestra una síntesis de los plaguicidas prohibidos o restringidos en Europa, permitidos en Chile y los efectos asociados en la salud humana $(3,43)$.

Entre 2010 y 2011 otro estudio, en la provincia de Talca(17), evaluó la exposición a plaguicidas OP y su asociación con factores de riesgos ambientales y sociodemográficos en 190 escolares de 6 a 12 años. La evaluación de la exposición se realizó a través de la medición de metabolitos OP en orina (DAPs) en dos periodos: verano (época de mayor exposición ambiental) y otoño (periodo de menor exposición ambiental). La presencia de metabolitos DAPs en la orina de escolares se asoció principalmente al consumo de frutas con clorpirifos y fosmet (manzanas, tomate y naranjas), vivir cerca de predios agrícolas y la aplicación de plaguicidas OP (principalmente fenitrotion) dentro del hogar.

Un reciente estudio, que evaluó en dos comunidades escolares rurales chilenas de la región del Maule(44), el efecto de una intervención para reducir la exposición a plaguicidas y midió antes y después la presencia de plaguicidas en la orina de niños y la percepción de riesgo de los mismos escolares y sus padres sobre lo plaguicidas, demostró que están expuestos a una gama de diversos tipos de agroquímicos que coincide con los plaguicidas más vendidos en la región. El estudio consideraba una intervención participativa de la comunidad con un grupo de intervención y otro de control, los escolares varones, de menor edad, que vivían y estudiaban a menos de 200 metros de predios agrícolas, presentaban mayor presencia de metabolitos OP en la orina. En esta investigación se encontró, en la mayoría de las orinas de los escolares, el metabolito paranitrofenol, asociado con el paration y metil paration. Ambos plaguicidas están prohibidos en Chile.

Tabla 1. Algunos plaguicidas organofosforados (OP) peligrosos, prohibidos o restringidos en Europa, vigentes en Chile y una síntesis de sus efectos.

\begin{tabular}{|c|c|c|c|c|c|c|}
\hline & $\begin{array}{c}\text { Toxicidad } \\
\text { aguda }\end{array}$ & cáncer & Mutagénico & $\begin{array}{c}\text { Disruptor } \\
\text { endocrino }\end{array}$ & $\begin{array}{c}\text { Afecta la } \\
\text { reproducción }\end{array}$ & $\begin{array}{c}\text { Daño } \\
\text { neurológico }\end{array}$ \\
\hline Azinfos Metil* & $\mathrm{X}$ & $\mathrm{X}$ & $\mathrm{X}$ & $\mathrm{X}$ & $\mathrm{X}$ \\
\hline Metamidofos* & $\mathrm{X}$ & $\mathrm{X}$ & $\mathrm{X}$ & & $\mathrm{X}$ & $\mathrm{X}$ \\
\hline Metidatión* & $\mathrm{X}$ & & & & $\mathrm{X}$ & $\mathrm{X}$ \\
\hline Malation & $\mathrm{X}$ & $\mathrm{X}$ & & & & $\mathrm{X}$ \\
\hline Clorpirifos & $\mathrm{X}$ & $\mathrm{X}$ & & & & $\mathrm{X}$ \\
\hline Dimetoato & $\mathrm{X}$ & $\mathrm{X}$ & & & $\mathrm{X}$ & \\
\hline Fenitrotión & $\mathrm{X}$ & $\mathrm{X}$ & & & & $\mathrm{X}$ \\
\hline Diazinon* & & & & & $\mathrm{X}$ \\
\hline
\end{tabular}

*Prohibido en Europa(49). 
Tanto los investigadores como las autoridades chilenas no logran explicar la presencia de este compuesto químico. Se manejan otras hipótesis, el analito paranitrofenol se asocia también con el nitrobenceno, compuesto que no está regulado en Chile, sí está prohibido en Europa y se encuentra principalmente en zonas mineras o en fertilizantes, esto último podría también explicar la presencia del metabolito en la orina; sin embargo, quedan las interrogantes sin resolver, al observar que en Argentina se vive una realidad similar, siendo más explícitos en mencionar que se aplican plaguicidas prohibidos mezclados con otros plaguicidas agrícolas(45).

Además, se encontró asociada la presencia de paratión con clorpirifos; sin embargo, no hay pruebas contundentes de la presencia del paratión en vegetales o en suelos, solo se ha encontrado en la orina de los escolares. Las consecuencias en la salud del paratión o del nitrobenceno son similares, afectan la fertilidad y, en condiciones de exposición crónica prolongada en el tiempo, puede generar cáncer(43).

Existe evidencias más que suficientes para levan- tar el principio precautorio, prohibir el uso de los plaguicidas más peligrosos aún vigentes en Chile (tabla 1), restringir la normativa, en cuanto a la venta y aplicación de plaguicidas, a personas capacitadas en su manipulación, solicitar el aumento de fiscalización en los predios agrícolas, análisis anuales de residuos de plaguicidas en las frutas y verduras de consumo nacional y de los plaguicidas que se aplican en el país, y vigilancia de metabolitos de plaguicidas en la población general, principalmente rural.

En dos estudios, realizados en Argentina, se encontró plaguicidas similares al estudio chileno en frutas que consume la población(14) y en aguas poco profundas de predios agrícolas(45). Esto fue denunciado, a su vez, en un documental realizado durante la misma época sobre la masiva exposición a plaguicidas en diversos pueblos rurales, autodenominándose "pueblos fumigados"(46). La condición de exposición de escuelas rurales, aplicación de plaguicidas peligrosos sobre comunidades rurales, escaso conocimiento sobre aplicación de plaguicidas y poca fiscalización, es similar a la realidad chilena $(1,6)$.

Tabla 2: Ejemplo de clasificación de agentes cancerígenos de la IARC para plaguicidas y otros (IARC, 2018).

\begin{tabular}{|c|c|c|c|}
\hline $\begin{array}{l}\text { Grupo } 1 \\
\text { (Cancerígeno para seres } \\
\text { humanos) }\end{array}$ & $\begin{array}{l}\text { Grupo 2A (Probablemente } \\
\text { cancerígeno) }\end{array}$ & $\begin{array}{l}\text { Grupo 2B } \\
\text { (Posiblemente cancerígeno } \\
\text { para los seres humanos) }\end{array}$ & $\begin{array}{l}\text { Grupo } 3 \\
\text { (No se clasifica) }\end{array}$ \\
\hline \multicolumn{4}{|l|}{ Plaguicidas: } \\
\hline \multirow[t]{6}{*}{ Lindano } & DDT & Diclorvos & Carbaril \\
\hline & Diazinon & $\begin{array}{l}\text { 2,4-D (2,4-ácido } \\
\text { diclorofenoxiacético) }\end{array}$ & Permetrina \\
\hline & Glifosato & Parathion & Endrin \\
\hline & Malation & & \\
\hline & Captafol & & \\
\hline & Dieldrin & & \\
\hline \multicolumn{4}{|l|}{ Ejemplo de otros agentes: } \\
\hline $\begin{array}{l}\text { Etanol y bebidas } \\
\text { alcohólicas }\end{array}$ & $\begin{array}{l}\text { Petróleo refinado } \\
\text { (ocupacional) }\end{array}$ & Café (vejiga y tracto urinario) & Colesterol \\
\hline Arsénico & Malaria & Combustible diésel marinos & $\begin{array}{l}\text { Combustibles diésel } \\
\text { destilados (light) }\end{array}$ \\
\hline $\begin{array}{l}\text { Consumo de tabaco en } \\
\text { fumadores y no fumadores }\end{array}$ & $\begin{array}{l}\text { Plomo compuesto } \\
\text { inorgánico }\end{array}$ & Isopreno & Diazepan \\
\hline
\end{tabular}


Discusión. Una mirada desde la bioética y la justicia ambiental

El solo hecho de que se presenten algunos indicios de riesgo y daño irreversible en los seres vivos es básico para decidir que se proteja legalmente el bienestar, seguridad y salud de las personas y el medio ambiente. El principio precautorio permite dar respuestas a la incertidumbre de las evidencias que no están totalmente probadas, pero que, sin duda, si se espera una consecuencia, puede ser más grave que haberlo aplicado como un derecho que poseen las sociedades para protegerse del daño que la industria, los desastres naturales o los mismos seres humanos han provocado en la Tierra $(1,47)$.

Un elemento clave para entender esto es considerar las clasificaciones que presenta la IARC(18). La IARC revisa agentes que generan riesgo o químicos contaminantes, principalmente ambientales y ocupacionales, y los efectos en la salud para el desarrollo de cáncer, clasificando según metaanálisis estudios que evalúen riesgo del grupo de peligrosidad.

La IARC(16) clasifica en 5 grupos la evaluación de la evidencia científica y la posible asociación con el cáncer en seres humanos. En el caso de los plaguicidas, algunos ejemplos de clasificación, comparados con otros agentes serían los siguientes (tabla 2).

La evaluación que realiza la IARC, que clasifica a los plaguicidas en posibles cancerígenos, aún es acotada. Existe suficiente evidencia que demuestra que el clorpirifos, azinfos metil y metamidofos son cancerígenos y no han sido evaluados por la IARC $(3,43)$; sin embargo, algunos de los plaguicidas que sí se clasifican como probablemente cancerígenos aún están permitidos en Chile, como el Diazinón, Malation y 2,4-D. Los dos primeros organofosforados y el tercero —un herbicida muy usado en el país - fueron encontrados en la orina de los escolares de la Región del Maule(44). La comunidad europea los tiene restringidos o prohibidos, y existe evidencia de la frecuente presencia de diazinón en la fruta consumida(43).

El informe de ACHIPIA(42) muestra cómo, no solo el diazinón se presenta en la uva de mesa, excediendo los límites permitidos por el MINSAL(41), si no también otros plaguicidas tan complejos para la salud humana, como el metamidofos, uno de los más notificados en el informe y que también excede límites en hortalizas - como las lechugas - que son consumidas por la población.

Considerando todos estos elementos, se observa que el problema de la nocividad alimentaria u ambiental existe en nuestro país asociada al cultivo agrícola, y requiere de una reflexión bioética urgente que evalúe la responsabilidad de los tomadores de decisiones en regular la priorización de la producción a escala masiva; de la exacerbación de lo tecnocientífico(48) en el mundo rural por sobre el cuidado por la naturaleza y enfocada en el mercado exterior por sobre la calidad del consumo interno de los chilenos; y del derecho de recibir productos inocuos y controlar la exposición y aplicación de estos contaminantes en la población, pero que a la vez invite a la comunidad a conocer estas consecuencias y a participar de manera activa en la elaboración de propuestas que permitan mitigar los riesgos ambientales y de exposición a plaguicidas.

La bioética, en el contexto medio ambiental(49), tiene un rol fundamental en lo que se refiere a difundir y exigir justicia ambiental para las comunidades y naciones vulneradas en su derecho de vivir libres de contaminantes, y de la basura o gradiente química que deriva de las grandes industrias y que generan gases invernaderos o aspersión de químicos que provocan efectos en la salud y en los alimentos que consumen. En este aspecto, las comunidades se ven injustamente expuestas a estos agrotóxicos que aplican las industrias o son incentivados en su uso sin la debida educación a las mismas comunidades. En ese sentido, la justicia distributiva es clave para entender que no solo se debe exigir que no contaminen los poblados y sus recursos, si no también dar espacios para la participación de dichas comunidades en generar una propuesta, acorde a su cultura y sus realidades, que prevenga la exposición a pesticidas y otros contaminantes. En eso es clave que sean actores presentes en la generación de normativas, políticas y estrategias de intervención, que permitan el cambio y 
la posibilidad de vivir en un ambiente libre de contaminantes industriales y con derecho a una salud plena y al consumo de productos agrícolas limpios.

En una revisión anterior(1), se habla sobre la situación de uso y control de plaguicidas en Chile. Se cuestiona la situación de leyes, que aún está pendiente en el Congreso, sobre la prohibición de los plaguicidas más peligrosos que aun se aplican en Chile y que están prohibidos en la mayoría de las partes del mundo, y sobre la regulación hacia quienes aplican estos agrotóxicos. El año 2018 se logró dirigir las miradas a la situación de la Región del Maule, sumado a la intoxicación masiva(50) que vivenciaron 25 niños en ese mismo año en una escuela rural de esa misma región (una de las con mayor población rural en el país), que demuestra justamente el riesgo ambiental y la injusticia de tener que estudiar al frente de un predio agrícola que aplica plaguicidas.

Se percibe la ausencia de un espacio de reflexión, conocimiento, responsabilidad y de empoderamiento de las comunidades, del consumidor nacional y de las autoridades, en hacerse cargo de la problemática y no invisibilizarla con la idea de que, si se trata el tema de la contaminación por plaguicidas, se corre el riesgo de perder mercado de productos agrícolas(51), mientras toda la población, incluidos los grandes productores, consumen los alimentos contaminados adquiridos en el mercado nacional.

En este punto es clave revisar la Declaración de Tbilisi(52), que fortalece la presencia de la educación ambiental en los contextos escolares, promoviendo la sensibilización y el cambio de comportamientos y actitudes con un enfoque participativo comunitario acerca de las problemáticas ambientales, aplicando estrategias de diversas disciplinas, como las ciencias sociales, la ecología y las ciencias de la salud y el medioambiente, con el fin de generar conciencia en la comunidad y orientar hacia una sociedad con un pensamiento crítico frente al cuidado de la vida y el medioambiente, y con una visión de desarrollo sustentable $(51,52)$. El cambio y la justicia ambiental depende de ser conscientes de la desigualdad ambiental, de salud y calidad de vida, y, por último, de dar la oportunidad para hacerse cargo del problema y resolverlo desde una acción participativa y comprometida.

Más allá del principio de justicia, la justicia ambiental busca más que velar por la equidad frente a los más vulnerables y que se reciba un tratamiento justo y de calidad similar al de otras comunidades no vulnerables; la justicia ambiental apunta a hacer que las mismas comunidades exijan y gatillen la necesidad de una sociedad más igualitaria, que vele por el derecho a vivir en un medio ambiente limpio(53) y que, al mismo tiempo, respete su realidad cultural y cosmovisión de la vida y la naturaleza, integrándola a los nuevos desafíos que se manifiestan desde las sociedades complejas y la naturaleza.

\section{Agradecimientos}

Agradecemos a los docentes que nos apoyaron en este artículo del Diplomado en Discernimiento Bioético de la UCM, en especial al Dr. Marcelo Correa. El artículo fue financiado por un FONDECYT 11150784 y un REDES 180078.

\section{Referencias}

1. Muñoz-Quezada MT. Aspectos bioéticos en el control y aplicación de plaguicidas en Chile. Acta Bioethica 2011; 17:95104.

2. Villaamil EC, Bovi G, Nassetta. Situación actual de la contaminación por plaguicidas en Argentina. Rev Int Contam Ambie 2013; 29: 25-43.

3. Alavanja M, Bonner M. Occupational pesticide exposures and cancer risk. A review. J Toxicol Environ Health B 2012; 15: 238-263.

4. Muñoz-Quezada MT, Lucero BA, Iglesias VP, Muñoz MP, Cornejo CA, Achu E, et al. Chronic exposure to organophosphate (OP) pesticides and neuropsychological functioning in farm workers: a review. Int J Occup Environ Health 2016; 22: 68-79.

5. Grillo A, Achú E, Muñoz-Quezada MT, Lucero B. [Exposure to organophosphate pesticides and peripheral polyneuropathy in workers from Maule region, Chile]. Rev Esp Salud Pública 2018; 92: 1-10. 
6. Muñoz-Quezada MT, Lucero BA, Iglesias VP, Muñoz MP, Cornejo CA, Achú E, et al. Exposure to organophosphate (OP) pesticides and health conditions in agricultural and non-agricultural workers from Maule, Chile. In J Environ Health 2016; 22: 68-79.

7. Ueker ME, Silva VM, Moi GP, Pignati WA, Mattos IE, Silva AMC. Parenteral exposure to pesticides and occurence of congenital malformations: hospital-based case-control study. BMC Pediatrics 2016; 16. DOI:10.1186/s12887-016-0667-x

8. Hernández-Mariano JÁ, Torres-Sánchez L, Bassol-Mayagoitia S, Escamilla-Nuñez MC, Cebrian ME, Villeda-Gutiérrez ÉA, et al. Effect of exposure to p,p' -DDE during the first half of pregnancy in the maternal thyroid profile of female residents in a Mexican floriculture area. Environmental Research 2017; 156: 597-604. DOI: 10.1016/j.envres.2017.04.013

9. Cremonese C, Piccoli C, Pasqualotto F, Clapauch R, Koifman RJ, Koifman S, et al. Occupational exposure to pesticides, reproductive hormone levels and sperm quality in young Brazilian men. Reproductive Toxicology 2017; 67: 174-185. DOI: 10.1016/j.reprotox.2017.01.001

10. Raines N, González M, Wyatt C, Kurzrok M, Pool C, Lemma T, et al. Risk factors for reduced glomerular filtration rate in a Nicaraguan community affected by Mesoamerican nephropathy. MEDICC Rev. 2014; 16(2): 16-22. PMID: 24878645.

11. Vazquez Boucard C, Lee-Cruz L, Mercier L, Ramírez Orozco M, Serrano Pinto V, Anguiano G, Cazares L, Díaz D. A study of DNA damage in buccal cells of consumers of well- and/or tap-water using the comet assay: Assessment of occupational exposure to genotoxicants. Environ Mol Mutagen. 2017; 58(8): 619-627. DOI: 10.1002/em.22111.

12. Delcour I, Spanoghe P, Uyttendaele M. Literature review: impact of climate change on pesticide use. Food Res Int. 2015; 68: 7-15.

13. Muñoz-Quezada MT, Lucero B, Iglesias V, Muñoz MP. [Exposure pathways to pesticides in schoolchildren in the Province of Talca, Chile]. Gac Sanit. 2014; 28: 190-195.

14. Mac Loughlin T, Peluso ML, Etchegoyen MA, Alonso LL, de Castro MC, Percudani MC, et al. Pesticides residues in fruits and vegetables of the argentine domestic market: ocurrence and quality. Food Control 2018; 93: 129-138.

15. Dragpharma. Sinpulkill collar gato. 2018. Disponible en: http://www.dragpharma.cl/producto.html/producto.id/1057/ detalle-especie.index/17

16. International Agency for Research on Cancer. Monographs on the identification of carcinogenic hazards to humans, 2018. Disponible en: https://monographs.iarc.fr/

17. Muñoz-Quezada MT, Iglesias V, Lucero B, Steenland K, Barr DB, Levy KR, et al. Predictors of exposure to organophosphate pesticides in schoolchildren in the province of Talca, Chile. Environ Int. 2012; 47: 28-36.

18. Naeher L, Tulve N, Egeghy P, Barr D, Adetona O, Fortmann R, Needham L, Bozeman E, Hilliard A, Sheldon L. Organophosphorus and pyrethroid insecticide urinary metabolite concentrations in young children living in a southeastern United State city. Sci Total Environ. 2010; 408: 1145-53.

19. Sierra-Diaz E, Celis de la Rosa AJ, Lozano-Kasten F, Trasande L, Peregrina-Lucano AA, Sandoval-Pinto E, et al. Urinary pesticide levels in children and adolescents residing in two agricultural communities in Mexico. Int J Environ Res Public Health 2019; 16: 2-8.

20. Lu C, Barr DB, Pearson MA, Waller LA. Dietary intake and its contribution to longitudinal organophosphorus pesticide exposure in urban/suburban children. Environ Health Perspect. 2008; 116(4): 537-542.

21. Valcke M, Samuel O, Bouchard M, Dumas P, Belleville D, Tremblay C. Biological monitoring of exposure to organophosphate pesticides in children living in peri-urban areas of the Province of Quebec, Canada. Int Arch Occup Environ Health 2006; 79: 568-77.

22. Curwin BD, Hein MJ, Sanderson WT, Nishioka MG, Reynolds SJ, Ward EM, et al. Pesticide contamination inside farm and nonfarm homes. J Occup Environ Hyg. 2005; 2(7): 357-367.

23. Lu C, Kedan G, Fisker-Andersen J, Kissel JC, Fenske RA. Multipathway organophosphorus pesticide exposures of preschool children living in agricultural and nonagricultural communities. Environ Res. 2004; 96(3): 283-289.

24. Luo Y, Zhang M. Multimedia transport and risk assessment of organophosphate pesticides and a case study in the northern San Joaquin Valley of California. Chemosphere 2009; 75(7): 969-978.

25. Van Wendel B, Mora AM, Lindh C, Hernández-Bonilla D, Córdoba L, Wesseling C, et al. Pesticide exposure and neurodevelopment in children aged 6-9 years from Talamanca, Costa Rica. Cortex 2016; 85: 137-150.

26. Sagiv S, Harris M, Gunier R, Kogut K, Harley K, Deardorff J, et al. Prenatal organophosphate pesticide exposure and traits related to autism spectrum disorders in a population living in proximity to agriculture. Environ Health Perspect 2018; 126: $1-9$.

27. Muñoz-Quezada MT, Lucero BA, Barr DB, Steenlad K, Levy K, Ryan PB, et al. Neurodevelopmental effects in children asociated with exposure to organophosphate pesticides: a systematic review. Neurotoxicology 2013; 39: 158-168.

28. Kalliora C, Mamoulaks C, Vasilopoulos E, Stamatiades G, Kalafati L, Barouni R, et al. Association of pesticide exposure with human congenital abnormalities. Toxicol Appl Pharmacol 2018; 346: 58-75. DOI: 10.1016/j.taap.2018.03.025.

29. Castillo-Cadena J, Mejia-Sanchez F, López-Arriaga JA. Congenital malformations according to etiology in newborns from the floricultural zone of Mexico State. Environmental Science and Pollution Research 2017; 24: 7662-7667. 
30. Bouchard MF, Chevrier J, Harley K, Kogut K, Vedar M, Calderon N, Trujillo C, Johnson C, Bradman A, Barr D, Eskenazi B. Prenatal exposure to organophosphates pesticides and IQ in 7-year-old children. Environ Health Perspect. 2011; 119(8): 1189-1195.

31. Eskenazi B, Marks AR, Bradman A, Harley K, Barr DB, Johnson C, et al. Organophosphate pesticide exposure and neurodevelopment in young Mexican-American children. Environ Health Perspect. 2007; 115(5): 792-798.

32. Ferreira JD, Couto AC, Pombo-De-Oliveira MDS, Koifman S. In utero pesticide exposure and leukemia in Brazilian children < 2 years of age. Environmental Health Perspec. 2013; 121(2): 269-275. DOI: 10.1289/ehp.1103942

33. Mei Y. Occupational exposure to pesticides towards the danger of childhood leukemia in China. Biomedical Reseach 2018; 29(1): 9-14.

34. Rojas A, Ojeda ME, Barraza X. Malformaciones congénitas y exposición a pesticidas. Rev Med Chil. 2000; 128(4): 399404.

35. Muñoz MT. Uso de plaguicidas y discapacidad intelectual en estudiantes de escuelas municipales, Provincia de Talca, Chile. Rev. Fac. Nac. Salud Pública. 2010; 28(1): 29-35.

36. Márquez C, Villalobos C, Poblete S, Villalobos E, García M, Duk S. Cytogenetic damage in female Chilean agricultural workers exposed to mixtures of pesticides. Environ Mol Mutagen. 2005; 45(1): 1-7.

37. Contreras-Levicoy J, Astorga E, Castro R, Yentzen G, Cumsille M. Abortos espontáneos en Hospital de Llay-Llay y su relación con labores agrícolas de la madre. Rev. Chil. Salud Pública 2005; 9(1): 7-11.

38. Zúñiga L, Garbiñe C, Palacios M. Estudio citogenético y reproductivo en mujeres temporeras expuestas a pesticidas de la VIII región de Chile. Theoria 2007; 16(1): 77-87.

39. Muñoz MT, Iglesias VP, Lucero BA. Exposición a organofosforados y desempeño cognitivo en escolares rurales chilenos: un estudio exploratorio. Rev. Fac. Nac. Salud Pública 2011; 29(3): 256-263.

40. SAG. Programa de monitoreo de residuos de plaguicidas en vegetales, año 2006. Santiago de Chile; 2007. Disponible en: http://www.conadecus.cl/documentos/1387_001.pdf

41. MINSAL. Fija tolerancias máximas de residuos de plaguicidas en alimentos y deja sin efecto la resolución exenta n 581 , de 1999, y sus modificaciones. Resolución exenta ${ }^{\circ} 33$. Santiago de Chile: Ministerio de Salud; 2010. Disponible en: http:// webhosting.redsalud.gov.cl/transparencia/public/minsal/normativa_a7c-2.html

42. ACHIPIA. Reporte de notificaciones 2017. Red de informacion y alertas alimentarias. Disponible en: https://www.achipia. gob.cl/wp-content/uploads/2019/02/OR.Informe-RIAL-2017-.pdf

43. UTZ. List of banned pesticides and pesticides watchlist, 2015. Available in: https://utz.org/wp-content/uploads/2015/12/EN_UTZ_List-of-Banned-PesticidesWatchlist_v1.0_2015.pdf

44. Muñoz-Quezada MT, Lucero B, Iglesias V, Muñoz MP, Zúñiga L, Concha C, et al. Effects of an educational intervention on organophosphate pesticides in the risk perception and chlorpyrifos, diazinon and parathion metabolites levels in chilean rural schoolchildren. Environ Health Pers 2018. Disponible en: https://ehp.niehs.nih.gov/doi/10.1289/isesisee.2018. O03.03.28

45. Mac Loughlin T, Peluso L, Marino D. Pesticide impact study in the peri-urban horticultural area of Gran La Plata, Argentina. Science Total Envi 2017; 598: 572-580.

46. Solanas F. Viaje a los pueblos fumigados. 2018. Disponible en: https://www.youtube.com/watch?v=M4kSHqWrdwI

47. Resnik D, Roman G. Salud, justicia y medio ambiente. Bioética 2007; 21: 230-241.

48. Zorrilla S. De una nocividad a la otra: la bioética frente al desafío de cultivar su pluralismo. Acta Bioethica 2001; 2: 317339.

49. Capó MA, Drane J. Planteamientos bioéticos del medio ambiente. Bioethikos 2014; 8: 46-52.

50. Costos de la agroexportación: nińos intoxicados por plaguicidas altamente peligrosos en Maule. Resumen 13 de octubre de 2018. Disponible en: https://resumen.cl/articulos/costos-de-la-agroexportacion-ninos-intoxicados-por-plaguicidas-altamente-peligrosos-en-maule

51. Lopes W, de Souza M. Pesticide use and economic impacts on health. Rev Saúde Pública 2012; 46: 1-8.

52. Sarmiento PJ. Bioética ambiental y ecopedagogía: una tarea pendiente. Acta Bioethica 2013; 19: 29-38.

53. Hervé D. Noción y elementos de la justicia ambiental: directrices para su aplicación en la planificación territorial y en la evaluación ambiental estratégica. Revista de Derecho 2010; 33: 9-36. 